\title{
Annealing Studies of irradiated p-type Sensors Designed for the Upgrade of ATLAS Phase-II Strip Tracker
}

\author{
L. Wiik-Fuchs ${ }^{\text {a,* }}$, L. Diehla ${ }^{\mathrm{a}}$, R. Mori ${ }^{\mathrm{a}}$, M.Hauser ${ }^{\mathrm{a}}$, K. Jakobs ${ }^{\mathrm{a}}$, S. Kühn ${ }^{\mathrm{a}}$, \\ U.Parzefall ${ }^{\mathrm{a}}$, A.A. Affolder ${ }^{\mathrm{d}}$, V. Fadeyev ${ }^{\mathrm{d}}$, C. García ${ }^{\mathrm{b}}$, C. Lacasta $^{\mathrm{b}}$, \\ D. Madaffari ${ }^{\mathrm{b}}$, U. Soldevila ${ }^{\mathrm{b}}$, Y. Unno ${ }^{\mathrm{c}}$ \\ ${ }^{a}$ Physikalisches Institut, Albert-Ludwigs-Universität Freiburg, Hermann-Herder-Str. 3, \\ 79104 Freiburg-im-Breisgau, Germany \\ ${ }^{b}$ Instituto de Física Corpuscular (IFIC) - CSIC-University of Valencia, Parque Científico, \\ C/Catedrático José Beltrán 2, E-46980 Paterna, Spain \\ ${ }^{c}$ IPNS, KEK, 1-1 Oho, Tsukuba, Ibaraki 305-0801, Japan \\ ${ }^{d}$ Santa Cruz Institute for Particle Physics (SCIPP), University of California, Santa Cruz, \\ CA 95064, USA
}

\begin{abstract}
The upgrade for the High Luminosity LHC in 2025 will challenge the silicon strip detector performance with high fluence and long operation time. Sensors have been designed and tests on charge collection and electrical performance have been carried out in order to evaluate their behaviour. Besides that, it is important to understand and predict the long-term evolution of the sensor properties. In this work, detailed studies on the annealing behaviour of ATLAS12 strip sensors designed by the ITK Strip Sensor Working Group and irradiated from $5 \times 10^{13} \mathrm{n}_{\mathrm{eq}} \mathrm{cm}^{-2}$ to $2 \times 10^{15} \mathrm{n}_{\mathrm{eq}} \mathrm{cm}^{-2}$ are presented. Systematic charge collection, leakage current and impedance measurements have been carried out during the annealing time at 23 and $60{ }^{\circ} \mathrm{C}$ until break-down or the appearance of charge multiplication. Sensors showing charge multiplication have been then kept at high voltage for a long time in order to monitor their stability. The difference in the annealing behaviour between the two temperatures has been analysed and compared to similar measurements on n-type sensors and with a theoretical model. From the impedance measurements for the samples irradiated to low fluences it was possible to extract the effective doping concentration. This was compared to similar measurements on n-type sensors and with a theoretical model. The results show that ATLAS12 sensors anneal similarly to the previously designed ATLAS07 and the behaviour is well described by the theoretical model. Nevertheless, a significant difference on the time constant of the beneficial and reverse annealing has been reported, especially at lower temperatures. For the highest fluences and longer annealing time, e.g. 5000 minutes at $60^{\circ} \mathrm{C}$, charge multiplication has been observed. The phenomenon is however temporary and disappears with the long-term voltage stress.
\end{abstract}

*L. Wiik-Fuchs e-mail: lwiik@mail.cern.ch 
Keywords:

\section{Introduction}

The high-luminosity LHC (HL-LHC) [1] will commence data-taking in 2025. With a total integrated luminosity of $4000 \mathrm{fb}^{-1}$ it will lead to unprecedented radiation fluences in the tracking layers, posing enormous challenges to the silicon detectors and their operation. Therefore the ATLAS collaboration [2] will replace the current Inner Detector with an all-silicon Inner Tracker [3]. The run schedule of the HL-LHC foresees shutdown periods on a yearly basis for machine maintenance. During part of these periods the silicon detectors can not be cooled. Hadron radiation leads to significant changes of the electrical properties of silicon sensors. These are caused by defects to the silicon bulk, which are subject to a complex migration process, called annealing and have a strong time and temperature dependence. Hence the annealing can be accelerated or decelerated by increasing or lowering the annealing temperature, as described by the Hamburg Model [4]. In general annealing studies are conducted at elevated temperatures, mostly at $60^{\circ} \mathrm{C}$, and measurements are then corrected to the equivalent of several years of room-temperature annealing. The Hamburg model is derived from measurements of n-bulk silicon, whereas p-bulk silicon sensors are mandatory at the HL-LHC, due to their superior radiation hardness $[5,6,7]$.

Therefore, a thorough understanding of the annealing behaviour of the silicon sensors used for the HL-LHC is mandatory for a successful planning of the operation scenarios. Previous studies on the annealing behaviour of p-type silicon can for instance be found in $[8,9,10,11]$. This study presents the annealing behaviour of ATLAS12 [12] sensors designed by the ATLAS Collaboration and manufactured by Hamamatsu Photonics [13]. The study includes ten sensors that were irradiated to five different fluences between $5 \times 10^{13} \mathrm{n}_{\mathrm{eq}} \mathrm{cm}^{-2}$ to $2 \times 10^{15} \mathrm{n}_{\mathrm{eq}} \mathrm{cm}^{-2}$. To assess the temperature dependence during the long term annealing, the sensors were separated into two groups and either annealed at room temperature or at $60^{\circ} \mathrm{C}$. After each annealing step, the sensor behaviour was evaluated measuring the charge collection and leakage current. Additional impedance measurements were done for a few sensors. Some sensors showed charge multiplication. They were further investigated in a long term stability measurement, applying a constant high bias voltage. However the results indicate that this effect is temporary and vanishes under long term operation at high bias voltages. Furthermore a detailed investigation on the difference in the annealing behaviour of sensors annealed at room-temperature and at $60^{\circ} \mathrm{C}$ are presented and a comparison to the Hamburg Model is given.

\section{Devices under Test}

The sensors evaluated in this study are miniature $(1 \mathrm{~cm} \times 1 \mathrm{~cm})$ sensors from the ATLAS12 prototype production, manufactured by Hamamatsu Photonics. These are single-sided p-bulk standard float-zone sensors with n-type implants, 
made in $\langle 100\rangle$ orientation. The sensors are cut from six inch wafers with a nominal thickness of $310 \pm 20 \mu \mathrm{m}$ and a thickness variation of less than $10 \mu \mathrm{m}$ within one sensor. The sensors have a bulk resistivity of about $3.5 \mathrm{k} \Omega$, hence the depletion voltage is on the order of $350 \mathrm{~V}$. Each miniature sensor has a strip pitch of $75.5 \mu \mathrm{m}$ such that each sensor contains 103 strips with a length of $0.8 \mathrm{~cm}$. The n-type strips are connected to the bias rail via poly-silicon resistors implanted in the sensor. Different interstrip isolation schemes are implemented, using either uniform p-spray, p-stop implants or a combination of both. This is to guarantee strip isolation during the lifetime of the sensors and further to weaken radiation-induced surface damage while maintaining a low inter-strip capacitance. Previous studies evaluating the performance of these sensors under irradiation are found in $[14,15,16]$.

To determine the sensor behaviour after exposure to radiation doses comparable to those expected at the HL-LHC, the sensors were irradiated using a $23 \mathrm{MeV}$ proton beam at the Irradiation Centre Karlsruhe. The evaluated sensors were irradiated to fluences of $5 \times 10^{13} \mathrm{n}_{\mathrm{eq}} \mathrm{cm}^{-2}, 1 \times 10^{14} \mathrm{n}_{\mathrm{eq}} \mathrm{cm}^{-2}$, $2 \times 10^{14} \mathrm{n}_{\mathrm{eq}} \mathrm{cm}^{-2}, 5 \times 10^{14} \mathrm{n}_{\mathrm{eq}} \mathrm{cm}^{-2}, 1 \times 10^{15} \mathrm{n}_{\mathrm{eq}} \mathrm{cm}^{-2}$ and $2 \times 10^{15} \mathrm{n}_{\mathrm{eq}} \mathrm{cm}^{-2}$.

\section{Experimental Setup}

Measurements of the collected charge were performed using a ${ }^{90} \mathrm{Sr}$ source, emitting MIP-like electrons. Triggering of particles traversing the detector is done using the coincidence signal of two scintillators placed behind the device under test. Using the coincidence of the two scintillators further helps to reject events arising from low energetic particles. The measurements are conducted in a freezer, flushed with nitrogen and are operated under temperature and humidity control. The measurements shown in this study were performed at temperatures in the range of $-14^{\circ} \mathrm{C}$ to $-17^{\circ} \mathrm{C}$ and a relative humidity of less than $10 \%$. The data acquisition is done using the ALiBaVa [17] system, which is based on LHC compatible $40 \mathrm{MHz}$ analogue electronics built around the Beetle chip [18]. The sensors were biased and the current was read out via a Keithley 237. The sensors annealed at $60^{\circ} \mathrm{C}$ were annealed in a dedicated temperature controlled oven with a temperature stability of $60 \pm 1^{\circ} \mathrm{C}$.

\section{Signal Analysis}

The charge collection measurements were taken at different bias voltages ranging from $100 \mathrm{~V}$ to $1100 \mathrm{~V}$. The signal was analysed using a customised software package, which includes an iterative common mode and pedestal subtraction algorithm. Additionally channels exhibiting an excessive noise behaviour were masked. Events were rejected if their trajectory did not originate from the path between the source and the scintillator area. Furthermore events are selected if they fall into a time window of $10 \mathrm{~ns}$ around the signal peak to allow for high statistics. This leads to a small signal underestimation of less than $5 \%$. 
The charge is determined using a clustering algorithm with a seed cut of 3.5 and a neighbouring cut of 1.8 times the noise value, to exclude spurious hits and noise contributions to the signal.

\section{Long Term Annealing Behaviour}

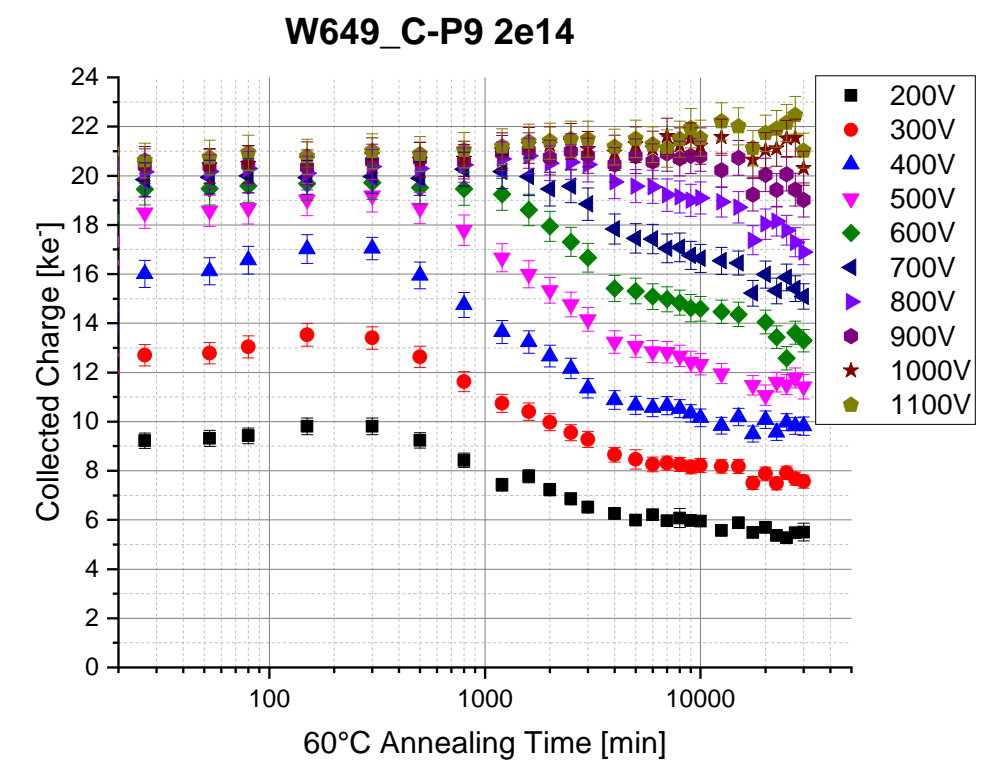

Figure 1: Most probable value of the collected charge of the sensor irradiated to $2 \times 10^{14} \mathrm{n}_{\mathrm{eq}} \mathrm{cm}^{-2}$ as a function of the annealing time for different bias voltages.

The initial charge collection measurement of the sensors was conducted without any additional annealing other than caused by transportation and handling of the sensors after irradiation. The duration of the annealing steps was increased from initially $30 \mathrm{~min}$ to $1000 \mathrm{~min}$ at $60^{\circ} \mathrm{C}$, to account for the logarithmic timescale of the behaviour. All sensors were annealed either until breakdown or charge multiplication occurred. The maximum annealing time is found for the sensor irradiated to $2 \times 10^{14} \mathrm{n}_{\mathrm{eq}} \mathrm{cm}^{-2}$, shown in Fig. 2 with more than $20000 \mathrm{~min}$ of accelerated annealing, corresponding to more than four years at room-temperature. The collected charge of the sensor follows the expected behaviour from the Hamburg model. First an increase of the collected charge is found for annealing times below $300 \mathrm{~min}$ attributed to the beneficial annealing. Thereafter the signal deteriorates for voltages below $800 \mathrm{~V}$, showing that the space charge region depth for the same voltage decreases with time in the so-called reverse annealing indicating the increase of the effective doping concentration. The increase of the full depletion voltage, due to reverse annealing, is 


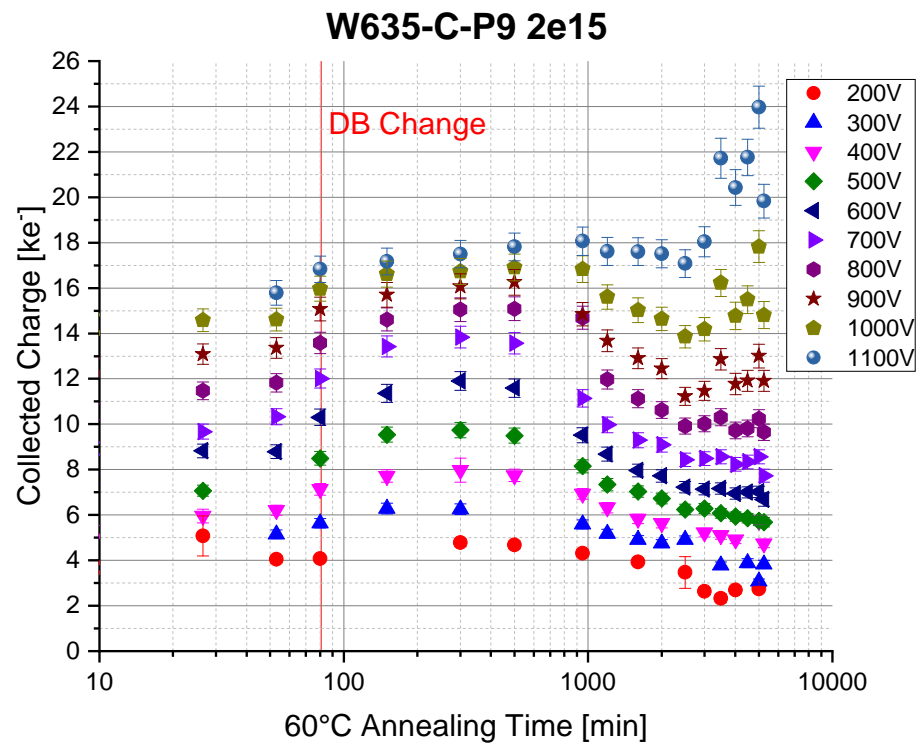

Figure 2: Most probable value of the collected charge of the sensor irradiated to $2 \times 10^{15} \mathrm{n}_{\mathrm{eq}} \mathrm{cm}^{-2}$ as a function of the annealing time for different bias voltages. DB change indicates the change between two daughterboards in the setup.However, given the external calibration, no difference to actual measurements are expected. 


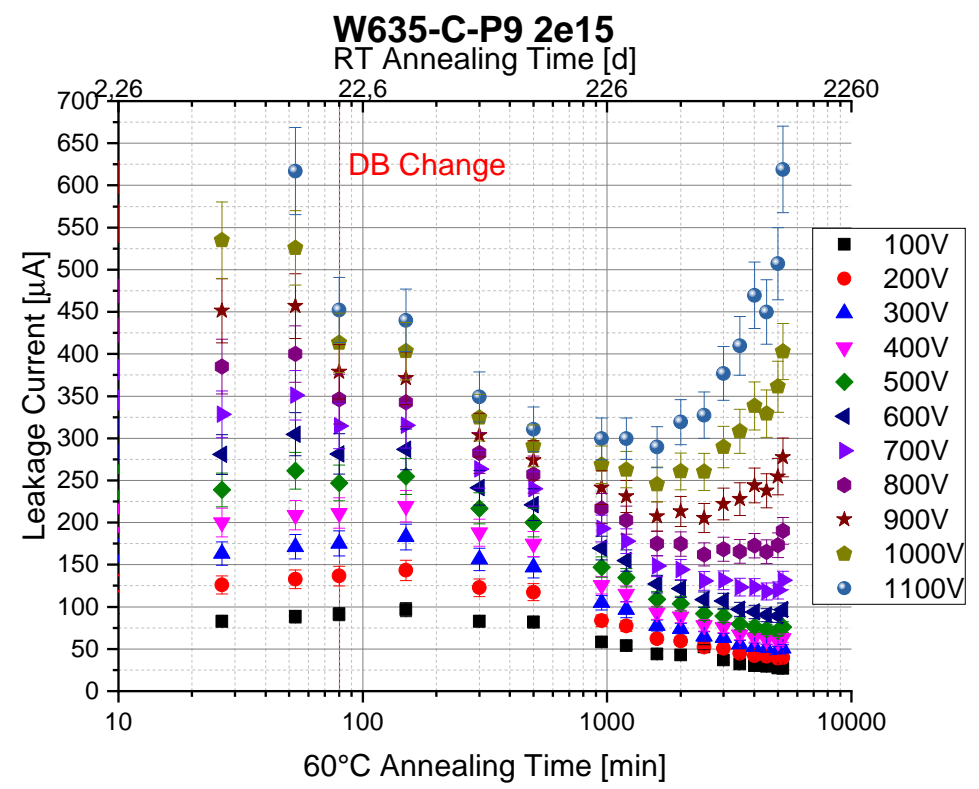

Figure 3: Leakage current of the sensor irradiated to $2 \times 10^{15} \mathrm{neq}_{\mathrm{eq}} \mathrm{cm}^{-2}$ as a function of the annealing time for different bias voltages. DB change indicates the change between two daughterboards in the setup.However, given the external calibration, no difference to actual measurements are expected 
seen from the increase in bias voltage needed for collecting the expected charge. No decline of the collected charge is seen for bias voltages higher than $1000 \mathrm{~V}$. In addition a slight increase of the collected charge for annealing times beyond $1000 \mathrm{~min}$ is seen. This might be attributed to the onset of charge multiplication, as suggested in previous studies [8].

Also for the sensor irradiated to $2 \times 10^{15} \mathrm{n}_{\mathrm{eq}} \mathrm{cm}^{-2}$, shown in Fig. 1, a clear increase of the collected charge due to beneficial annealing is seen in all voltages up to $300 \mathrm{~min}$ of annealing, followed by the expected decrease of the collected charge during the reverse annealing process. Annealing times beyond $3000 \mathrm{~min}$ lead to a strong increase of the collected charge for bias voltages above $900 \mathrm{~V}$. The increase is attributed to an annealing-induced charge multiplication. This interpretation is supported by the behaviour of the corresponding reverse current shown in Fig. 5. Whilst the reverse current decreases, as expected, during beneficial and reverse annealing a strong increase is seen for annealing times beyond $3000 \mathrm{~min}$ at high bias voltages in agreement to the occurrence of charge multiplication.

To evaluate the impact and the possible use of charge multiplication in the detector operation, a long term stability measurement was performed, keeping the sensor irradiated to $2 \times 10^{15} \mathrm{n}_{\mathrm{eq}} / \mathrm{cm}^{-2}$ and annealed for $5500 \mathrm{~min}$ at $60{ }^{\circ} \mathrm{C}$ at a constant bias voltage of $1100 \mathrm{~V}$, while recurrently measuring the collected charge and the reverse current. The collected charge as a function of the measuring time is shown in Fig. 1. Given the broadening of the signal due to the increased noise, both the most probable value (MPV) and the median were extracted from the fit to the signal distribution. However, both exhibit the same features. The initial most probable value of the collected charge was at $24 \mathrm{ke}^{-}$, which is consistent with the expected charge collection for an unirradiated sensor of this thickness. Under constant stress the collected charge of the sensor decreased until it reached a plateau after $390 \mathrm{~h}$ at a level of $10 \mathrm{ke}^{-}$. Thereafter the sensor underwent a further annealing step of $80 \mathrm{~min}$ at $60^{\circ} \mathrm{C}$. This lead to an even higher charge collection of $30 \mathrm{ke}^{-}$, however the signal decline was even stronger reaching a constant level of $12 \mathrm{ke}^{-}$after $100 \mathrm{~h}$ under constant stress at a bias voltage of $1100 \mathrm{~V}$. The sensor was then kept at rest at a temperature of $-15^{\circ} \mathrm{C}$ for another $17 \mathrm{~d}$, which again lead to a slight increase of the signal to $18 \mathrm{ke}^{-}$. Also this increase was not high voltage stable and decreased in the following measurements. The noise of the sensor was also recorded during the long term stress measurement and is shown in Fig. 4(b). A clear correlation between the collected charge and the noise is seen. A decrease from a noise level of $1.4 \mathrm{ke}^{-}$down to $1 \mathrm{ke}^{-}$is observed during the first $400 \mathrm{~h}$ of the long term high bias measurement. The additional annealing step lead to an increase of the noise beyond the initial starting value to $1.9 \mathrm{ke}^{-}$. Continuing the high biassing lead to an about four times faster decrease of the noise, such that a noise level of $1.1 \mathrm{ke}^{-}$is reached after $100 \mathrm{~h}$. Again storing the sensor without bias lead to an increase in the noise level, which thereafter drops below $0.8 \mathrm{ke}^{-}$.

The HL-LHC run schedule foresees a long shutdown on a yearly basis for machine maintenance. During at least part of these periods the silicon detectors 

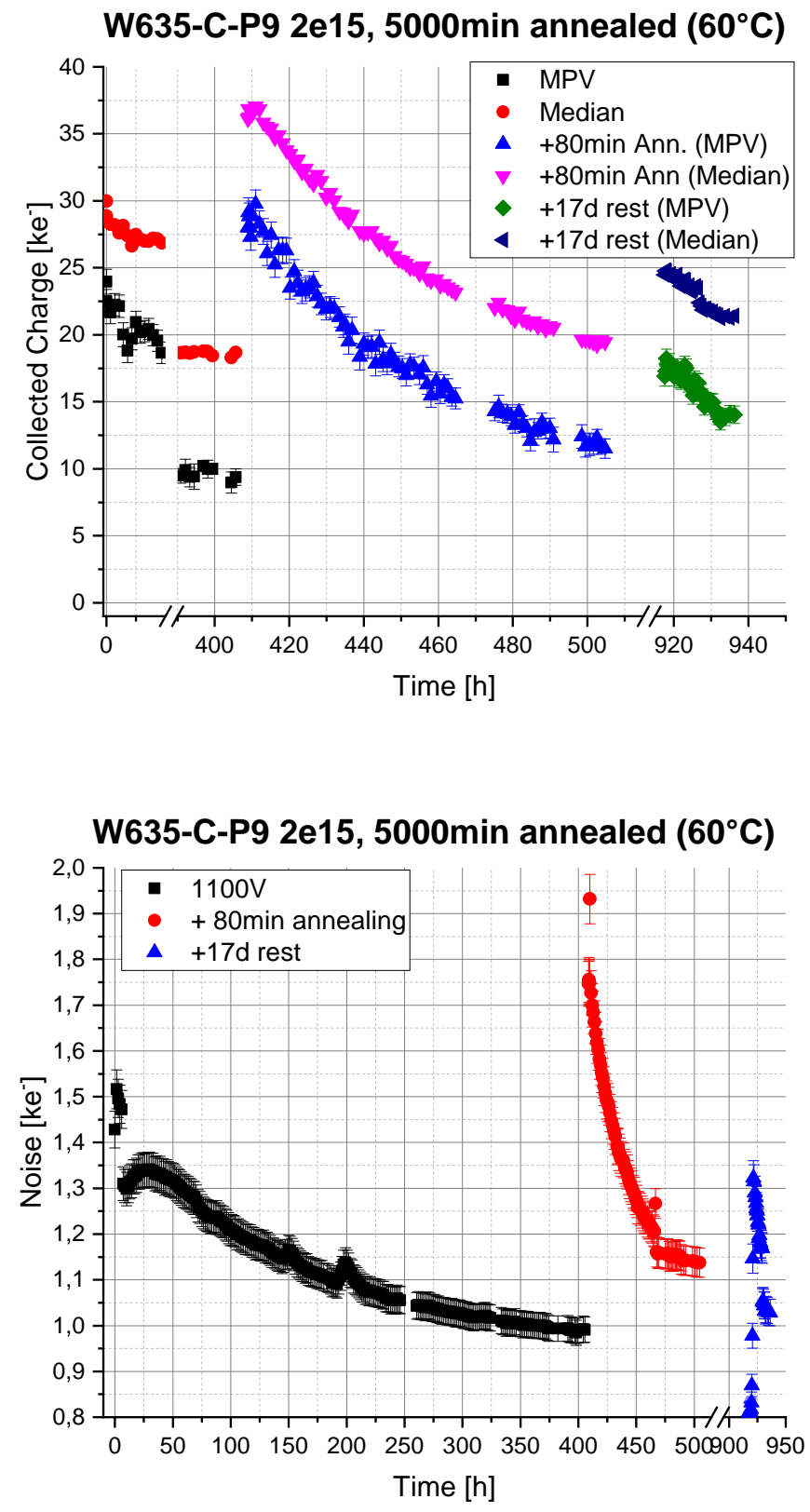

Figure 4: Long term stability measurement of the charge multiplication signal as a function of the measuring time. The upper figure shows the collected charge, whereas the lower figure shows the noise behaviour during the measurements. 

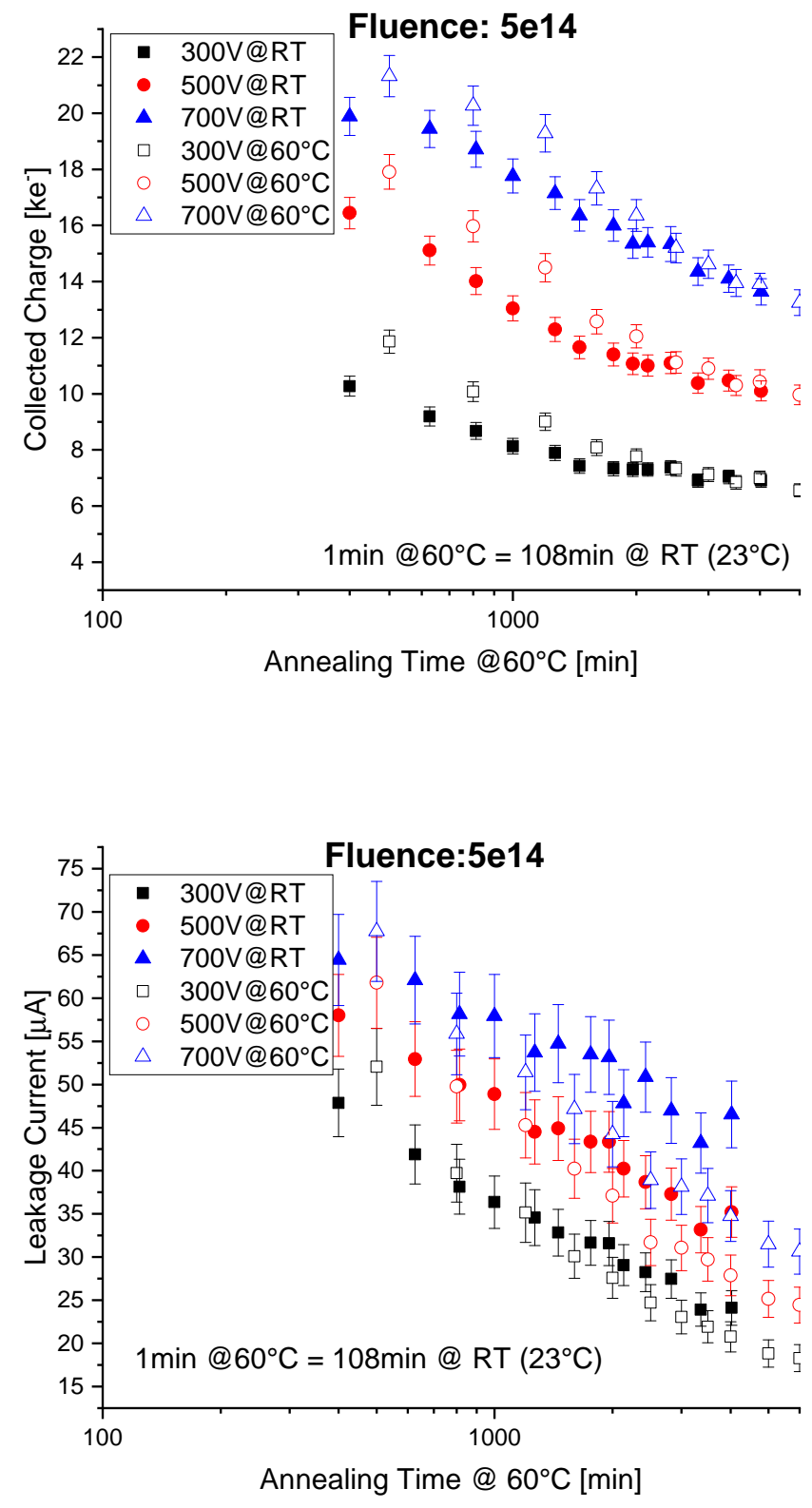

Figure 5: Charge collection measurements 5(a) and reverse current 5(b) for sensors irradiated to $5 \times 10^{14} \mathrm{n}_{\mathrm{eq}} \mathrm{cm}^{-2}$, taken at three distinctive bias voltages for room-temperature and accelerated annealing at $60^{\circ} \mathrm{C}$. The factor was determined by a graphical overlay of the corresponding distributions at the two temperatures. 


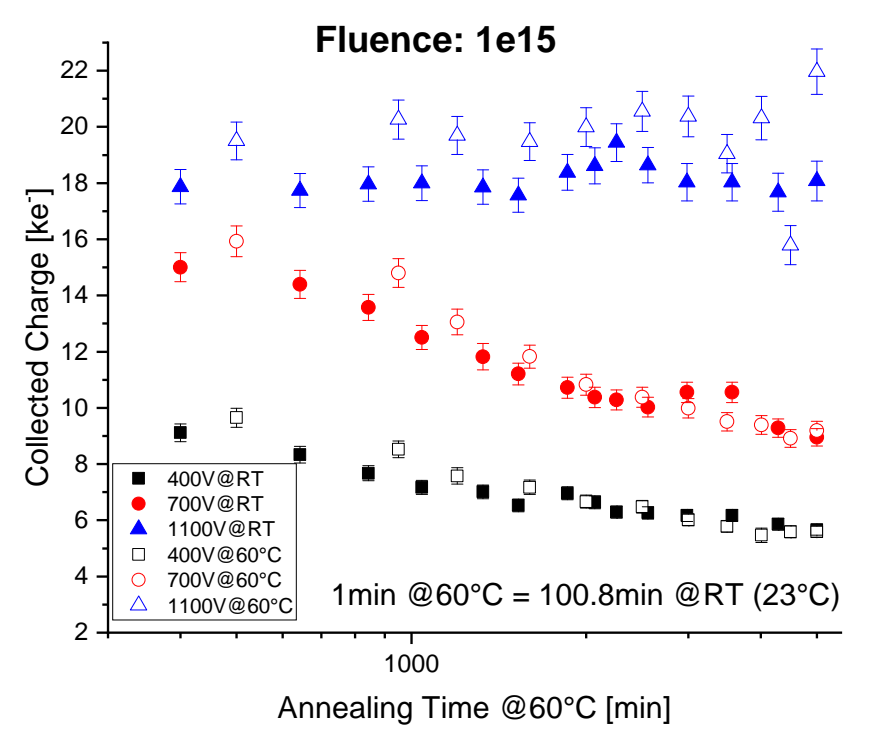

\section{Fluence: 1 e15}

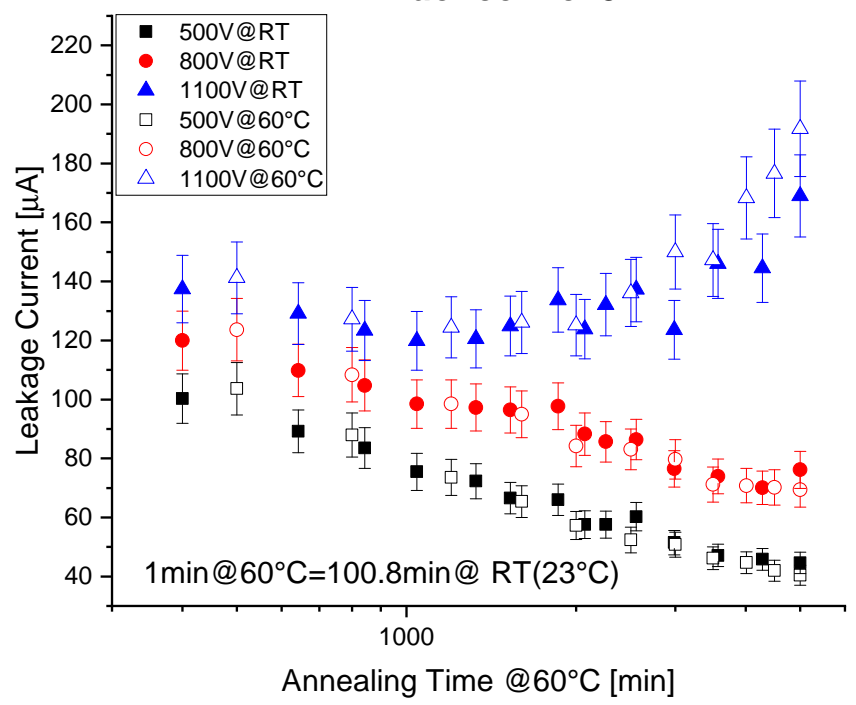

Figure 6: Charge collection measurements 6(a) and reverse current 6(b) for sensors irradiated to $1 \times 10^{15} \mathrm{n}_{\mathrm{eq}} \mathrm{cm}^{-2}$. Taken at three distinctive bias voltages for room-temperature and accelerated annealing at $60^{\circ} \mathrm{C}$. The factor was determined by a graphical overlay of the corresponding distributions at the two temperatures. 
Table 1: Factor between room-temperature and accelerated annealing at $60^{\circ} \mathrm{C}$. The factor was determined by a graphical overlay of the two distributions

\begin{tabular}{cc|cccc} 
Fluence & $5 \times 10^{13}$ & $1 \times 10^{14}$ & $5 \times 10^{14}$ & $1 \times 10^{15}$ & $2 \times 10^{15}$ \\
\hline Scaling Factor & $108(8)$ & $101(15)$ & $108(12)$ & $101(9)$ & $108(8)$
\end{tabular}

will likely be held at room-temperature of $23^{\circ} \mathrm{C}$ und thus undergo a certain annealing. The assumed annealing behaviour of the sensors during these periods is derived from accelerated annealing measurements taken at $60^{\circ} \mathrm{C}$ and scaled with a factor of 325. This factor is derived from the Arrhenius relation of the temperature dependence in the reverse annealing [19]. To verify this scaling ATLAS12 sensors irradiated to five different fluences between $5 \times 10^{13} \mathrm{n}_{\mathrm{eq}} \mathrm{cm}^{-2}$ to $2 \times 10^{15} \mathrm{n}_{\mathrm{eq}} \mathrm{cm}^{-2}$, were separated into two subsets annealed at either room temperature or at $60^{\circ} \mathrm{C}$. The behaviour of the sensors was evaluated in terms of charge collection, reverse current and impedance measurements during the annealing process. Data for more than a year of room temperature annealing was collected. The charge collection and the reverse current as a function of the annealing time is shown in Figure 5 for the sensor irradiated to $5 \times 10^{14} \mathrm{n}_{\mathrm{eq}} \mathrm{cm}^{-2}$ and in Fig. 6 for the sensor irradiated to $1 \times 10^{15} \mathrm{n}_{\mathrm{eq}} \mathrm{cm}^{-2}$. The scaling factor between room temperature and $60^{\circ} \mathrm{C}$ annealing is determined using an iterative graphical overlay approach. Especially the trend in the leakage current shows a good agreement between the two temperatures at all voltages. The factors determined for all the different fluences are summarised in Tab. 1 and are on the order of 105 [19]. This is roughly a factor three smaller than the expected literature value. In particular, measuring the onset of the reverse annealing it seems that the sensors studied here have a slower annealing at higher temperatures, rather then a faster annealing at lower temperatures. The disparity between the factors may be attributed to a different oxygen concentration in the bulk material, the use of p-type silicon compared with n-type silicon or further changes to the detector properties. The study is currently being extended, by additionally determining the effective doping concentration from impedance measurements, which are supporting above results and by annealing the sensors at other temperatures.

\section{Conclusion}

The operation conditions expected at the HL-LHC will challenge the silicon strip detectors both with high fluences and long operation times. A thorough understanding of the detector behaviour under these conditions mandatory for a successful data taking. Therefore a detailed study of the annealing behaviour of ATLAS12 sensors, irradiated with $23 \mathrm{MeV}$ protons to fluences ranging from $5 \times 10^{13} \mathrm{n}_{\text {eq }}$ to $2 \times 10^{15} \mathrm{n}_{\mathrm{eq}}$ has been carried out. Annealing-induced changes to the sensor behaviour were evaluated by measuring the charge collection, impedance and leakage current in increasing time intervals of annealing. Measurements were taken until either a break-down of the sensor or charge multiplication occurred. Sensors showing charge multiplication, were subjected to long 
term stability measurements, applying a constant high bias voltage.

Recurrent charge collection measurements revealed that the increased signal is not stable and vanishes over time. Further annealing or resting of the sensor without bias voltage, leads to a significant recovery of the charge multiplication signal, however the following deterioration of the signal is even accelerated. This shows that operating sensors in radiation induced charge multiplication is not encouraging for stable long-term operations.

Investigations on the temperature dependence during the long term annealing behaviour were made, annealing sensors either at room temperature or at $60{ }^{\circ} \mathrm{C}$. The results indicate that ATLAS12 sensors anneal similarly to the previously designed ATLAS07 [20] and the behaviour generally follows the Hamburg model. A significant difference was found for the acceleration factor. While it is expected to be 325 according to [19] and [8], the measurements presented here indicate that it is a factor of three smaller. Hence the ATLAS12 sensors anneal faster at room temperature than predicted and a thorough understanding of the room-temperature annealing is crucial for detector operations.

\section{Acknowledgements}

The research was supported and financed in part by USA Department of Energy, Grant DE-SC0010107; European Union's Horizon 2020 Research and

Innovation programme under Grant Agreement no. 654168. The authors would like to thank Alexander Dierlamm for the proton irradiations at the Karlsruhe Institute of Technology (KIT), supported by the Initiative and Networking Fund of the Helmholtz Association, contract HA-101(Physics at the Terascale)and the European Commission under the FP7 Research Infrastructures project AIDA, Grant agreement no.262025.

\section{References}

[1] A. G. and et al., High-Luminosity Large Hadron Collider (HL-LHC): Technical Design Report V. 0.1. CERN Yellow Reports: Monographs. CERN, Geneva, 2017. https://cds.cern.ch/record/2284929.

[2] ATLAS Collaboration, The ATLAS Experiment at the CERN Large Hadron Collider, Journal of Instrumentation 3 no. 08, (2008) S08003. http://stacks.iop.org/1748-0221/3/i=08/a=S08003.

[3] ATLAS Collaboration, Technical Design Report for the ATLAS Inner Tracker Strip Detector, Tech. Rep. CERN-LHCC-2017-005.

ATLAS-TDR-025, CERN, Geneva, Apr, 2017. https://cds.cern.ch/record/2257755.

[4] ROSE Collaboration, G. Lindstrom et al., Radiation hard silicon detectors developments by the RD 48 (ROSE) Collaboration, Nucl. Instrum. Meth. A466 (2001) 308-326. 
[5] G. Casse, P. Allport, and M. Hanlon, Improving the radiation hardness properties of silicon detectors using oxygenated n-type and p-type silicon, IEEE Trans. Nucl. Sci. 47 no. 3, (2000) 527-532.

http://dx.doi.org/10.1109/23.856475.

[6] G. Kramberger, V. Cindro, I. Mandic, M. Mikuz, and M. Zavrtanik, Influence of trapping on silicon microstrip detector design and performance, IEEE Trans. Nucl. Sci. 49 (2002) 1717-1723.

[7] V. Cindro, G. Kramberger, M. Lozano, I. Mandic, M. Mikuz, G. Pellegrini, J. Pulko, M. Ullan, and M. Zavrtanik, Radiation damage in p-type silicon irradiated with neutrons and protons, Nucl. Instrum. Meth. A599 (2009) 60-65.

[8] I. Mandić, V. Cindro, G. Kramberger, and M. Mikuž, Annealing effects in $n+-p$ strip detectors irradiated with high neutron fluences, Nuclear Instruments and Methods in Physics Research Section A: Accelerators, Spectrometers, Detectors and Associated Equipment 629 no. 1, (2011) $101-105$. http://www.sciencedirect.com/science/article/pii/S0168900210025209.

[9] G. Casse, P. P. Allport, and A. Watson, Effects of accelerated annealing on p-type silicon micro-strip detectors after very high doses of proton irradiation, Nucl. Instrum. Meth. A568 (2006) 46-50.

[10] G. Kramberger, M. Batic, V. Cindro, I. Mandic, M. Mikuz, and M. Zavrtanik, Annealing studies of effective trapping times in silicon detectors, Nucl. Instrum. Meth. A571 (2007) 608-611.

[11] M. Minano, J. P. Balbuena, C. Garcia, S. Gonzalez, C. Lacasta, V. Lacuesta, M. Lozano, S. Marti i Garcia, G. Pellegrini, and M. Ullan, Annealing studies of silicon microstrip detectors irradiated at high neutron fluences, Nucl. Instrum. Meth. A591 (2008) 181-183.

[12] Y. Unno et al., Development of $n^{+}$-in-p large-area silicon microstrip sensors for very high radiation environments - ATLAS12 design and initial results, Nucl. Instrum. Meth. A765 (2014) 80-90.

[13] Hamamatsu Photonics K.K., 1126-1 Ichino-cho, Hamamatsu-shi 435-8558, Japan,http://www.hamamatsu.com, 2016. http://www.hamamatsu.com.

[14] M. Mikestikova and et al., Study of surface properties of ATLAS12 strip sensors and their radiation resistance, Nuclear Instruments and Methods in Physics Research Section A: Accelerators, Spectrometers, Detectors and Associated Equipment 831 (2016) 197 - 206.

http://www.sciencedirect.com/science/article/pii/S0168900216300730. Proceedings of the 10th International "Hiroshima" Symposium on the Development and Application of Semiconductor Tracking Detectors. 
[15] K.Hara and et al., Charge collection and field profile studies of heavily irradiated strip sensors for the ATLAS inner tracker upgrade, Nuclear Instruments and Methods in Physics Research Section A: Accelerators, Spectrometers, Detectors and Associated Equipment 831 (2016) 181 188.

http://www.sciencedirect.com/science/article/pii/S0168900216302078. Proceedings of the 10th International "Hiroshima" Symposium on the Development and Application of Semiconductor Tracking Detectors.

[16] R. Mori and et al., Evaluation of the performance of irradiated silicon strip sensors for the forward detector of the ATLAS Inner Tracker Upgrade, Nuclear Instruments and Methods in Physics Research Section A: Accelerators, Spectrometers, Detectors and Associated Equipment 831 (2016) $207-212$.

http://www.sciencedirect.com/science/article/pii/S0168900216302522. Proceedings of the 10th International "Hiroshima" Symposium on the Development and Application of Semiconductor Tracking Detectors.

[17] R. Marco-Hernández, A portable readout system for silicon microstrip sensors, Nuclear Instruments and Methods in Physics Research Section A: Accelerators, Spectrometers, Detectors and Associated Equipment 623 no. 1, (2010) 207-209. https://doi.org/10.1016/j.nima.2010.02.197.

[18] S. Löchner and M. Schmelling, The Beetle Reference Manual - chip version 1.3, 1.4 and 1.5,

[19] M. Moll, Radiation damage in silicon particle detectors: Microscopic defects and macroscopic properties. PhD thesis, University of Hamburg, Germany, 1999.

http://www-library.desy.de/cgi-bin/showprep.pl?desy-thesis99-040.

[20] Y. Unno and et al., Development of n-on-p silicon sensors for very high radiation environments, Nuclear Instruments and Methods in Physics Research Section A: Accelerators, Spectrometers, Detectors and Associated Equipment 636 no. 1, (2011) S24-S30. http://dx.doi.org/10.1016/j.nima.2010.04.080. 